\title{
Flat free boundaries regularity in two-phase problems for a class of fully nonlinear elliptic operators with variable coefficients
}

\author{
ROBERTO ARgiolas \\ Dipartimento di Matematica e Informatica dell'Università di Cagliari, \\ viale Merello 92/94, 09123 Cagliari, Italy \\ E-mail: roberarg@unica.it \\ FAUSTO FERRARI \\ Dipartimento di Matematica dell'Università di Bologna, \\ Piazza di Porta San Donato, 5, 40126 Bologna, Italy \\ and C.I.R.A.M., Via Saragozza 8, 40123 Bologna, Italy \\ E-mail:ferrari@dm.unibo.it
}

[Received 26 July 2007 and in revised form 25 July 2008]

We study the regularity of the free boundary in two-phase problems for fully nonlinear elliptic operators. In particular, we prove that flat free boundaries are $C^{1, \gamma}$.

\section{Introduction and main results}

In this paper we prove that flat free boundaries of the solutions of elliptic two-phase problems associated with a class of fully nonlinear operators are $C^{1, \gamma}$. In [11] the $C^{1, \gamma}$ regularity of Lipschitz free boundaries of two-phase problems was proved for a class of homogeneous fully nonlinear elliptic operators $F\left(D^{2} u(x), x\right)$, containing convex (concave) operators, with Hölder dependence on $x$. Here we consider the same class of operators. More precisely, we prove the regularity of flat free boundaries of the solutions of the following two-phase problems:

$$
\begin{cases}F\left(D^{2} u(x), x\right)=0 & \text { in } \Omega^{+}(u)=\left\{x \in \Omega \subset \mathbb{R}^{n}: u>0\right\} \\ F\left(D^{2} u(x), x\right)=0 & \text { in } \Omega^{-}(u)=\left\{x \in \Omega \subset \mathbb{R}^{n}: u \leqslant 0\right\}^{\circ} \\ u=0 & \text { on } \mathcal{F}_{u}, \\ u_{v}^{+}=G\left(u_{v}^{-}\right) & \text {on } \mathcal{F}_{u},\end{cases}
$$

where $\mathcal{F}_{u}=\partial \Omega^{+} \cap \Omega$ is the free boundary, and $u^{ \pm} \in C(\Omega)$ is a viscosity solution of the equation $F\left(D^{2} u(x), x\right)=0$ respectively in $\Omega^{+}(u)$ and $\Omega^{-}(u)$.

We denote by $B_{r}\left(x_{0}\right)$ the ball in $\mathbb{R}^{n}$ centered at $x_{0}$ with radius $r$. Hence (see [1]), the conditions on the free boundary $\mathcal{F}_{u}$ are satisfied in the following sense: if $x_{0} \in \mathcal{F}_{u}$ and there exists a ball $B_{\rho}(y)$ such that $x_{0} \in \partial B_{\rho}(y)$ and $B_{\rho}(y)$ is contained either in $\Omega^{+}(u)$ or in $\Omega^{-}(u)$, then

$$
u(x)=\alpha\left\langle x-x_{0}, v\right\rangle^{+}-\beta\left\langle x-x_{0}, v\right\rangle^{-}+o\left(\left|x-x_{0}\right|\right) \quad \text { as } x \rightarrow x_{0},
$$

where $v$ is the unit normal to $\partial B_{\rho}(y)$ at $x_{0}$ pointing to $\Omega^{+}(u)$ and

$$
\alpha=G(\beta) .
$$


Moreover, we assume that the function $G(z)$ is continuous, strictly increasing and for some $N>0$, $z^{-N} G(z)$ is decreasing in $(0,+\infty)$.

We say that free boundaries are flat whenever they are contained in an $\varepsilon$-neighborhood of some Lipschitz continuous graphs (see Theorem 1.1). Here we solve the problem of the regularity of flat free boundaries of the solutions of problem (1) assuming a nondegeneracy condition on the positive part of the solution. Roughly speaking, we are going to consider solutions $u$ such that $u^{+} / \operatorname{dist}\left(x, \mathcal{F}_{u}\right)$ behaves like a constant in a neighborhood of the free boundaries (see Theorem 1.1 (i) for the precise condition).

Under these hypotheses we can prove a sort of weak monotonicity of the solutions, the so-called $\varepsilon$-monotonicity (see Definition 3.2), which plays a key role in the proof of the regularity of the free boundaries. In this note we follow the main ideas contained in [2] and [16] adapting the approach used in [13] for the linear case with variable coefficients, where the notion of strict $\varepsilon$-monotonicity was introduced.

Regularity of the free boundaries has been studied following a geometric approach since the papers by Luis Caffarelli ([1], [2]) for the Laplace operator. Successively these results were extended to fully nonlinear operators with constant coefficients (see [9], [15], [10] and [16]), and to linear and nonlinear operators with variable coefficients (see [6], [11], [12] and [13]).

We are now going to introduce a class of fully nonlinear operators for our free boundaries problems.

Let $\mathcal{S}$ be the space of $n \times n$ symmetric matrices. Let $\mathcal{P}^{+}$and $\mathcal{P}^{-}$be the Pucci extremal operators on the class $\mathcal{A}_{\lambda, \Lambda}$ of symmetric matrices with lowest eigenvalue not smaller than $\lambda>0$ and highest eigenvalue not bigger than $\Lambda$ (see Section 2 for the definitions).

Concerning our class of fully nonlinear operators, we assume that $F: \mathcal{S} \times \Omega \rightarrow \mathbb{R}$ in (1) satisfies the following conditions:

(a) For all $M, N \in \mathcal{S}$ and $x, y \in \Omega$,

$$
\begin{aligned}
\mathcal{P}^{-}(M- & N, \lambda, \Lambda)-\omega(|x-y|)(\|M\|+\mid N \|) \\
& \leqslant F(M, x)-F(N, y) \leqslant \mathcal{P}^{+}(M-N, \lambda, \Lambda)+\omega(|x-y|)(\|M\|+\|N\|),
\end{aligned}
$$

where $\omega$ is a nondecreasing continuous function satisfying $\omega(0)=0$, and $\|M\|$ denotes the $\left(L^{2}, L^{2}\right)$-norm of $M \in \mathcal{S}$ (i.e. $\left.\|M\|=\sup _{|x|=1}|M x|\right)$. In particular $F$ is a fully nonlinear uniformly elliptic operator (see [3]).

(b) The operator $F$ is positive homogeneous of degree 1, i.e.

$$
F(\alpha M, x)=\alpha F(M, x)
$$

for all $\alpha>0, M \in \mathcal{S}$ and every $x \in \Omega$.

(c) $F$ has $C^{1,1}$ interior estimates: there exists a positive constant $c_{e}$ such that for every $x_{0} \in \Omega$, every $r>0$ such that the ball $B_{r}\left(x_{0}\right)$ is contained in $\Omega$, and every $w \in C\left(\partial B_{r}\left(x_{0}\right)\right)$, there exists a solution $\tilde{h} \in C^{2}\left(B_{r}\left(x_{0}\right)\right) \cap C\left(\bar{B}_{r}\left(x_{0}\right)\right)$ of the Dirichlet problem

$$
\begin{cases}F\left(D^{2} \tilde{h}(x), x_{0}\right)=0 & \text { in } B_{r}\left(x_{0}\right), \\ \tilde{h}(x)=w & \text { on } \partial B_{r}\left(x_{0}\right),\end{cases}
$$

and this solution $\tilde{h}$ satisfies the estimate

$$
\|\tilde{h}\|_{C^{1,1}\left(B_{r / 2}\left(x_{0}\right)\right)} \leqslant c_{e} r^{-2}\|\tilde{h}\|_{L^{\infty}\left(B_{r}\left(x_{0}\right)\right)} .
$$


We observe that the condition (c) replaces, in a sense, convexity (concavity) assumptions on $F$ in order to ensure, with the further hypothesis of (Lipschitz) regularity of the boundary of $\Omega$ and of $\left(C^{\alpha}\right)$ dependence on $x$, the existence and uniqueness of $L^{p}$-viscosity solutions $u \in W_{\text {loc }}^{2, p}(\Omega) \cap$ $C(\bar{\Omega})$ of the Dirichlet problem for $F$ in $\Omega$ (see [4]). Moreover, such hypotheses ensure interior $W^{2, p}$ estimates for $u$ (see [3], [4] and [14]). Indeed, under hypotheses (a)-(c), the Dirichlet problem

$$
\begin{cases}F\left(D^{2} \tilde{h}(x), x\right)=0 & \text { in } D, \\ \tilde{h}(x)=w & \text { on } \partial D\end{cases}
$$

admits a unique $L^{p}$-viscosity solution $\tilde{h} \in W_{\text {loc }}^{2, p}(D) \cap C(\bar{D})$ in a domain $D$ satisfying a uniform exterior cone condition when there exists a constant $M=M(n, p, \lambda, \Lambda)$ such that

$$
\left(\frac{1}{r^{n}} \int_{B_{r}\left(x_{0}\right)} \beta\left(x, x_{0}\right)^{p} \mathrm{~d} x\right)^{1 / p} \leqslant M, \quad r \leqslant r_{0},
$$

where

$$
\beta\left(x, x_{0}\right)=\sup _{M \in \mathcal{S}} \frac{\left|F(M, x)-F\left(M, x_{0}\right)\right|}{1+\|M\|}
$$

is the oscillation of $F$ in the variable $x$. In our case

$$
\beta\left(x, x_{0}\right) \leqslant \sup _{M \in \mathcal{S} \backslash\{0\}} \frac{\left|F(M, x)-F\left(M, x_{0}\right)\right|}{\|M\|} \leqslant 2 \omega\left(\left|x-x_{0}\right|\right)
$$

so that condition (3) holds if $\omega(s)=C s^{a}$, and $a$ is a fixed number, $a \in(0,1]$.

Let now $B_{r}^{\prime}$ be the ball in $\mathbb{R}^{n-1}$ centered at 0 with radius $r$. We define $\mathcal{C}_{r}=B_{r}^{\prime} \times(-r, r)$. The main result of this paper can be summarized in the following theorem.

THEOREM 1.1 Let $u$ be a solution of the free boundary problem (f.b.p., for short) (1) in $\mathcal{C}_{1}$ for $F(M, x)$ satisfying (a)-(c) and assume that $\omega(s)=\bar{C} s^{a}$ with $a \in(0,1]$ and $\bar{C}>0$ fixed. Suppose moreover that:

(i) there exist positive numbers $\alpha_{0}, \alpha_{1}$ such that

$$
\alpha_{0} \leqslant \frac{u^{+}(x)}{\operatorname{dist}\left(x, \mathcal{F}_{u}\right)} \leqslant \alpha_{1},
$$

(ii) $G(0)>0, G$ is a Lipschitz continuous function, strictly increasing in $\mathbb{R}^{+}$and, for some large constant $N, s^{-N} G(s)$ is decreasing.

Then there exist $\bar{\vartheta} \in(0, \pi / 2)$ and $\bar{\varepsilon}>0$ such that if for some $\varepsilon \in(0, \bar{\varepsilon})$ the set $\mathcal{F}_{u}$ is contained in the $\varepsilon$-neighborhood of the graph of a Lipschitz function $h, x_{n}=h\left(x^{\prime}\right)$, with Lipschitz norm

$$
\operatorname{Lip}(h) \leqslant \tan (\pi / 2-\bar{\vartheta})
$$

then in $B_{1 / 2}^{\prime} \subset \mathbb{R}^{n-1}, h$ is a $C^{1, \gamma}$ function with $\gamma=\gamma\left(n, a, \bar{C}, \alpha_{0}, \alpha_{1}, \lambda, \Lambda, N, \operatorname{Lip}(h)\right)$.

We always assume that $F(M, \cdot)$ satisfies hypotheses (a)-(c) and moreover that $\omega(s)=\bar{C} s^{a}$ with $a \in(0,1]$ and $\bar{C}>0$ fixed. Hence, we shall not repeat those assumptions in the following 
statements even if, sometimes, we shall stress the dependence of the constants on the several parameters involved in problem (1).

Concerning the notation, the various constants $c, C$, etc. that will appear may vary from formula to formula. In any case, if for such constants we do not give any explicit dependence, we mean that they depend on some of the relevant parameters $n, a, \bar{C}, \lambda, \Lambda, L, N$.

\section{Preliminaries}

For $M \in \mathcal{S}$, we define Pucci's extremal operators as

$$
\begin{aligned}
& \mathcal{P}^{-}(M, \lambda, \Lambda)=P^{-}(M)=\lambda \sum_{e_{i}>0} e_{i}+\Lambda \sum_{e_{i}<0} e_{i}, \\
& \mathcal{P}^{+}(M, \lambda, \Lambda)=P^{+}(M)=\lambda \sum_{e_{i}<0} e_{i}+\Lambda \sum_{e_{i}>0} e_{i},
\end{aligned}
$$

where $e_{i}=e_{i}(M)$ are the eigenvalues of $M$. Let now $A$ be a symmetric matrix whose eigenvalues belong to $[\lambda, \Lambda]$, i.e. $\lambda|\xi|^{2} \leqslant A_{i j} \xi_{i} \xi_{j} \leqslant \Lambda|\xi|^{2}$ for any $\xi \in \mathbb{R}^{n}$. We will then write $A \in \mathcal{A}_{\lambda, \Lambda}$. Define a linear functional $L_{A}$ on $\mathcal{S}$ by

$$
L_{A} M=\operatorname{Tr}(A M)
$$

It is known (see [3]) that

$$
\mathcal{P}^{-}(M, \lambda, \Lambda)=\inf _{A \in \mathcal{A}_{\lambda, \Lambda}} L_{A} M, \quad \mathcal{P}^{+}(M, \lambda, \Lambda)=\sup _{A \in \mathcal{A}_{\lambda, \Lambda}} L_{A} M .
$$

In this paper we deal with $C$-viscosity solutions of problem (1). Nevertheless in order to prove some monotonicity properties (see Lemma 3.1, we still apply some results concerning $L^{p}$-viscosity solutions. For this reason we recall both notions and we refer to [3], [4] and [14] for further details.

Definition $2.1 u \in C(\Omega)$ is a $C$-viscosity subsolution of $F\left(D^{2} u(x), x\right)=0$ in $\Omega$ if for every $x_{0} \in \Omega$ and every test function $\varphi \in C^{2}\left(\mathbb{R}^{n}\right)$, whenever $u-\varphi \leqslant u\left(x_{0}\right)-\varphi\left(x_{0}\right)$ in a neighborhood of $x_{0}$, then

$$
F\left(D^{2} \varphi\left(x_{0}\right), x_{0}\right) \geqslant 0
$$

analogously $u$ is a $C$-viscosity supersolution of $F\left(D^{2} u(x), x\right)=0$ in $\Omega$ if for every $x_{0} \in \Omega$ and every test function $\varphi \in C^{2}\left(\mathbb{R}^{n}\right)$, whenever $u-\varphi \geqslant u\left(x_{0}\right)-\varphi\left(x_{0}\right)$ in a neighborhood of $x_{0}$, then

$$
F\left(D^{2} \varphi\left(x_{0}\right), x_{0}\right) \leqslant 0 ;
$$

$u$ is a $C$-viscosity solution if it is both a $C$-viscosity subsolution and $C$-viscosity supersolution.

Let $p$ be such that $2 p>n$, and let $h \in L_{\mathrm{loc}}^{p}(\Omega)$.

DEFINITION 2.2 A function $u \in C(\Omega)$ is an $L^{p}$-viscosity subsolution (respectively, supersolution) of $F\left(D^{2} u(x), x\right)=h(x)$ in $\Omega$ if, for all $\varphi \in W_{\text {loc }}^{2, p}(\Omega)$, whenever $\varepsilon>0, D \subset \Omega$ is open and

$$
F\left(D^{2} \varphi(x), x\right)-h(x) \leqslant \varepsilon \quad \text { a.e. in } D
$$

(respectively, $F\left(D^{2} \varphi(x), x\right)-h(x) \geqslant-\varepsilon$ a.e. in $D$ )

then $u-\varphi$ cannot have a local maximum (respectively, minimum) in $D ; u$ is an $L^{p}$-viscosity solution if it is both an $L^{p}$-viscosity subsolution and $L^{p}$-viscosity supersolution. 
REMARK 2.1 We observe that if $u$ is a viscosity solution of $F\left(D^{2} u(x), x\right)=0$ (in one of the two senses), then $u_{r}(x)=u(r x)$ is a viscosity solution of $F_{r}\left(D^{2} u_{r}(x), x\right):=F\left(D^{2} u(r x), r x\right)=0$.

Let $T_{s}=\left\{\left(x^{\prime}, x_{n}\right) \in \mathbb{R}^{n}:\left|x^{\prime}\right|<s, f\left(x^{\prime}\right)<x_{n}<2 L s\right\}$, where $f$ is a Lipschitz function with Lipschitz norm $L$. For every positive $r$ and for every positive constant $M$ we set $Q_{M}=(1 / r) T_{M r}$. The boundary Harnack theorem for fully nonlinear operators can be found in [8] (see also Lemma 2.1 in [15] and Proposition 2.2 in [10]). In particular, we state here the so called boundary (Harnack) comparison theorem with its main corollary.

Proposition 2.1 Let $u, v$ be two nonnegative $L^{p}$-viscosity solutions of $F\left(D^{2} u(x), x\right)=0$ in $T_{2}$ and let $u, v$ vanish on $\left\{x_{n}=f\left(x^{\prime}\right)\right\} \cap \partial T_{2}$. Suppose that $u \geqslant \sigma v$ in $T_{2}$ for some $\sigma \geqslant 0$. Then

1) There exists $C=C(n, L)>0$ such that in $T_{1 / 2}$,

$$
C \frac{u(A)-\sigma v(A)}{v(A)} \leqslant \frac{u-\sigma v}{v}
$$

where $A=(L / 2) e_{n}$.

2) If in addition $u(A)=v(A)$ then $u / v \in C^{\alpha}\left(\bar{T}_{1 / 2}\right)$, where $\left.\alpha \in\right] 0,1[$.

In the case of operators independent of $x$ the proof can be found in [10, Proposition 2.2]. We omit the proof for operators depending on $x$ enjoying conditions (a) and (b), since it repeats the same argument used in [10].

\section{3. $\varepsilon$-Monotonicity and full monotonicity}

LEMMA 3.1 Let

$$
T_{s}=\left\{\left(x^{\prime}, x_{n}\right) \in \mathbb{R}^{n}:\left|x^{\prime}\right|<s, f\left(x^{\prime}\right)<x_{n}<2 L s\right\}
$$

where $f$ is a Lipschitz function with constant $L$. Let $u$ be a positive solution of $F\left(D^{2} u(x), x\right)=0$ in $T_{4}$, vanishing on $\Sigma=\left\{x_{n}=f\left(x^{\prime}\right)\right\} \cap T_{4}$. Then there exists $\eta=\eta(n, a, \bar{C}, \lambda, \Lambda, L)>0$ such that, in

$$
\mathcal{N}_{\eta}(\Sigma)=\left\{f\left(x^{\prime}\right)<x_{n}<f\left(x^{\prime}\right)+\eta\right\} \cap T_{1},
$$

$u$ is increasing along the directions $\tau$ belonging to the cone $\Gamma\left(e_{n}, \vartheta\right)$ with axis $e_{n}$ and opening $\vartheta=\frac{1}{2} \cot ^{-1} L$. Moreover, in $\mathcal{N}_{\eta}(\Sigma)$,

$$
c^{-1} \frac{u(x)}{d_{x}} \leqslant D_{n} u(x) \leqslant c \frac{u(x)}{d_{x}}
$$

where $d_{x}=\operatorname{dist}(x, \Sigma)$ and $c=c(n, a, L, \lambda, \Lambda)$.

Proof. Let $z$ be the solution of the following Dirichlet problem, associated with the operator $F(\cdot, x)$ frozen at $x_{0} \in T_{1 / 2}$ :

$$
\begin{cases}F\left(D^{2} z(x), x_{0}\right)=0 & \text { in } B_{2 R \varepsilon} \\ z=w_{r} & \text { on } \partial B_{2 R \varepsilon}\end{cases}
$$

where

$$
w_{r}(x)=\frac{u(r x)}{r}
$$


For simplicity let us assume that $x_{0}=0$. Then

$$
F\left(D^{2} w_{r}(x), r x\right)-F\left(D^{2} z(x), r x\right)=\operatorname{Tr}\left(A(x, r) D^{2}\left(w_{r}(x)-z(x)\right)\right)
$$

where

$$
A(x, r)=\left(a_{i j}(x, r)\right)_{1 \leqslant i, j \leqslant n} \in L^{\infty}\left(B_{2 R \varepsilon}\right)
$$

and

$$
a_{i j}(x, r)=\int_{0}^{1} \frac{\partial F}{\partial m_{i j}}\left(D^{2} z(x)+t D^{2}\left(w_{r}(x)-z(x)\right), r x\right) \mathrm{d} t .
$$

Moreover,

$$
F\left(D^{2} z(x), 0\right)-F\left(D^{2} z(x), r x\right)=f_{r}(x)
$$

and

$$
\left|f_{r}(x)\right| \leqslant 2 \omega(|x|)\left|D^{2} z(x)\right| .
$$

We set $h_{r}=w_{r}-z$. Then $h_{r}$ satisfies the equation

$$
\operatorname{Tr}\left(A(x, r) D^{2} h_{r}\right)=f_{r}(x) \quad \text { in } T^{\sigma} \cap B_{2 R \varepsilon}
$$

where

$$
T^{\sigma}=\left\{x \in T_{2}: \operatorname{dist}\left(x, \partial T_{2}\right)>\sigma\right\} .
$$

Now by the Aleksandrov-Bakelman-Pucci maximum principle, the $W^{2, p}$ estimate on $z$ and the boundary Harnack principle $]^{1}$ (see [8], [10] and [11]), we find, arguing as in [11, Lemma 2.5], that in any ball $B_{\sigma}(\bar{y})$ such that $B_{4 \sigma}(\bar{y}) \subset T^{\sigma}$ and $\operatorname{dist}\left(\bar{y}, \partial T^{\sigma}\right) \leqslant C \sigma$,

$$
\sup _{\partial B_{\sigma}(\bar{y})}\left|h_{r}\right| \leqslant c \sigma^{a} \max _{B_{\sigma}(\bar{y})} z \leqslant c \sigma^{a} z(\bar{y}) \quad \text { and } \sup _{B_{\sigma}(\bar{y})}\left|h_{r}\right| \leqslant c\left(\sigma^{a}+\omega(r)\right) z(\bar{y}) .
$$

On the other hand, $h_{r}$ is the solution of the equation

$$
F\left(D^{2} v, r x\right)=F\left(D^{2} h_{r}, r x\right) \in L^{p},
$$

because

$$
\mathcal{P}^{-}\left(-D^{2} z(x)\right) \leqslant F\left(D^{2} h_{r}, r x\right)=F\left(D^{2} h_{r}, r x\right)-F\left(D^{2} w_{r}, r x\right) \leqslant \mathcal{P}^{+}\left(-D^{2} z(x)\right) .
$$

Let $v$ be a viscosity solution of $F\left(D^{2} v, x\right)=f \in L^{p}$. Then the following $W^{2, p}$ estimate on $v$ holds:

$$
\sigma^{2-n / p}\left\|D^{2} v\right\|_{L^{p}\left(B_{\sigma}(\bar{y})\right)}+\sigma^{1-n / p}\|D v\|_{L^{p}\left(B_{\sigma}(\bar{y})\right)} \leqslant C\left(\|v\|_{L^{\infty}\left(B_{2 \sigma}(\bar{y})\right)}+\sigma^{2-n / p}\|f\|_{L^{p}\left(B_{2 \sigma}(\bar{y})\right)}\right)
$$

(see [7], [3] and [14]). In particular, by the Sobolev theorem we know that $h_{r} \in C^{1, \alpha}$ and

$$
\sigma\left|D h_{r}\right|_{C^{1}\left(B_{\sigma}(\bar{y})\right)} \leqslant C\left(\left\|h_{r}\right\|_{L^{\infty}\left(B_{2 \sigma}(\bar{y})\right)}+\sigma^{2-n / p}\left\|F\left(D^{2} h_{r}, r x\right)\right\|_{L^{p}\left(B_{2 \sigma}(\bar{y})\right)}\right) .
$$

As a consequence, from (5) keeping in mind the $W^{2, p}$ estimates on $z$ we get

$$
\left\|f_{r}\right\|_{L_{\left(B_{2 \sigma}(\bar{y})\right)}^{p}} \leqslant C \omega(r) z(\bar{y}) .
$$

\footnotetext{
1 The proof of the boundary Harnack principle holds with minor changes for this class of operators.
} 
In particular, we deduce, recalling the $W^{2, p}$ estimates of $h_{r}$ as an $L^{p}$-viscosity solution of 44 (see [4]), that

$$
\left|D h_{r}\right|_{C^{1}\left(B_{\sigma}(\bar{y})\right)} \leqslant C \omega(r) \frac{z(\bar{y})}{\sigma} .
$$

On the other hand (see [10] and [11]), there exist $c_{1}$ and $c_{2}$ such that

$$
c_{1} \frac{z(\bar{y})}{d_{\bar{y}}} \leqslant D_{n} z(\bar{y}) \leqslant c_{2} \frac{z(\bar{y})}{d_{\bar{y}}} .
$$

As a consequence there exist positive constants $c, C$ such that

$$
(1-c \omega(r)) D_{n} z(\bar{y}) \leqslant D_{n} w_{r}(\bar{y}) \leqslant(1+C \omega(r)) D_{n} z(\bar{y}) .
$$

Hence there exists $r_{0}$ such that $D_{n} w_{r}(\bar{y}) \geqslant 0$ for every $r \leqslant r_{0}$. The rest of the proof follows the techniques applied in [11, Lemmas 2.6 and 2.8] (see also steps (3) and (4) of Theorem 2.1 in [13]).

A key notion in the regularity of flat free boundary is $\varepsilon$-monotonicity.

DEFINITION 3.1 A function $u$ is $\varepsilon$-monotone in a domain $D$, along a direction $\tau$, if

$$
u\left(x+\varepsilon^{\prime} \tau\right)-u(x) \geqslant 0
$$

for every $x \in D$ and every $\varepsilon^{\prime} \geqslant \varepsilon$.

Lemma 3.2 and Corollary 3.1 below were proved in [2] (see also Section 5 in [5]). They depend only on purely geometric considerations and continue to hold in our context.

LEMMA 3.2 Let $u$ be $\varepsilon$-monotone in the cone $\Gamma(\vartheta, e)$. Let

$$
v(x)=\sup _{y \in B_{g(x)}(x)} u(y)
$$

where $g$ is a positive smooth function. Suppose that, for every $x$ in question,

$$
\sin \bar{\vartheta} \leqslant \frac{1}{1+|\nabla g|}\left(\sin \vartheta-\frac{\varepsilon}{2 g} \cos ^{2} \vartheta-|\nabla g|\right) .
$$

Then $v$ is monotone in the cone $\Gamma(\bar{\vartheta}, e)$.

COROLLARY 3.1 For any $\varepsilon$-monotone function $u$ in the cone $\Gamma(\vartheta, e)$, the level surfaces of $u$, $\partial\{u>\alpha\}$, are contained in the $(1-\sin \vartheta) \varepsilon$-neighborhood of the graph of a Lipschitz function $\tilde{h}$. In particular, denoting by $L^{\prime}$ the Lipschitz norm of $\tilde{h}$, if $\bar{\vartheta}$ satisfies 3.1 then $L^{\prime} \leqslant \cot \bar{\vartheta}$.

Analogously to the linear case with variable coefficients (see [13]), we need to introduce a slightly stronger notion than $\varepsilon$-monotonicity.

DEFINITION 3.2 A nonnegative function $u$ is strictly $\varepsilon$-monotone increasing with constant $\lambda>0$ in a domain $D$, along a direction $\tau$, if

$$
u\left(x+\varepsilon^{\prime} \tau\right)-u(x) \geqslant \lambda \varepsilon u(x)
$$

for every $x \in D$ and every $\varepsilon^{\prime} \geqslant \varepsilon$. 
Accordingly, a nonpositive function $u$ is strictly $\varepsilon$-monotone increasing if $u^{-}$is strictly $\varepsilon$ monotone decreasing, i.e. $u^{-}\left(x+\varepsilon^{\prime} \tau\right)-u^{-}(x) \leqslant-\lambda \varepsilon u^{-}(x)$. Finally, $u$ is strictly $\varepsilon$-monotone if $u^{+}$and $u^{-}$are strictly $\varepsilon$-monotone increasing and decreasing, respectively.

The next result shows that strictly $\varepsilon$-monotone solutions of our f.b.p. are indeed fully monotone $\varepsilon$-away from the free boundary as long as the coefficients are very close to being constant. This is the situation one finds after a suitable initial blow up centered at a free boundary point. Precisely, we have:

LEMMA 3.3 Let $u$ be a positive viscosity solution of $F\left(D^{2} u(x), x\right)=0$ in $B_{4 R \varepsilon}=B_{4 R \varepsilon}(0)$ such that

$$
u\left(x+\varepsilon^{\prime} \tau\right)-u(x) \geqslant \lambda \varepsilon u(x)
$$

in $B_{2 R \varepsilon}$, for some $\lambda>0$ and every $\varepsilon^{\prime} \geqslant \varepsilon$. There exists a positive number $\bar{m}=\bar{m}(p, n, a)$ such that for every $m \geqslant \bar{m}+4$, then there exists $R=R(n)$ and positive constants $C, c$ such that if $\varepsilon^{(m-2) /(\bar{m}+2)} \leqslant c \lambda$ and

$$
\|\omega(|x|)\|_{\infty} \leqslant C \varepsilon^{m}
$$

then

$$
D_{\tau} u(0) \geqslant c \lambda \frac{u(\varepsilon \tau)-u(0)}{\varepsilon} .
$$

Proof. Let $z$ be the solution of

$$
\begin{cases}F\left(D^{2} z(x), 0\right)=0 & \text { in } B_{2 R \varepsilon} \\ z=w_{r} & \text { on } \partial B_{2 R \varepsilon}\end{cases}
$$

where, as in Lemma 3.1. we define $w_{r}(x)=u(r x) / r$. Proceeding now as in Lemma 3.1, we have

$$
\begin{aligned}
F\left(D^{2} w_{r}(x), r x\right)-F\left(D^{2} z(x), r x\right) & =\operatorname{Tr}\left(A(x, r) D^{2}\left(w_{r}(x)-z(x)\right)\right), \\
F\left(D^{2} z(x), 0\right)-F\left(D^{2} z(x), r x\right) & =f_{r}(x),
\end{aligned}
$$

and

$$
\left|f_{r}(x)\right| \leqslant 2 \omega(|x|)\left|D^{2} z(x)\right| .
$$

We set $h_{r}=w_{r}-z$. Thus $h_{r}$ satisfies the following problem:

$$
\begin{cases}\operatorname{Tr}\left(A(x, r) D^{2} h_{r}\right)=f_{r}(x) & \text { in } B_{2 R \varepsilon}, \\ h_{r}=0 & \text { on } \partial B_{2 R \varepsilon} .\end{cases}
$$

Now by the Aleksandrov-Bakelman-Pucci maximum principle we get, whenever $\rho<1$,

$$
\sup _{B_{2 \rho \varepsilon R}}\left|h_{r}\right| \leqslant \max _{\partial B_{2 \rho \varepsilon R}}\left|h_{r}\right|+\omega(r)(2 \rho \varepsilon R)^{2-n / p}\left\|D^{2} z\right\|_{L^{p}\left(B_{2 \rho \varepsilon R}\right)} .
$$

On the other hand, if $\sigma=1-\rho$ then by the boundary Harnack principle,

$$
\max _{\partial B_{2 \rho \varepsilon R}}\left|h_{r}\right| \leqslant c(2 \sigma \varepsilon R)^{a} z(0) .
$$


We can cover $B_{2 \rho \varepsilon R}$ with balls centered at $x_{i}$ with radius $r_{i}=c \sigma \varepsilon R$ such that $B_{r_{i}} \subset B_{2 \varepsilon R}$. We need $N$ balls. Moreover, $N \sim c(\rho / \sigma)^{n}$. On each ball we can apply the $W^{2, p}$ estimate on $z$ (see also Lemma 3.1). Thus

$$
\begin{aligned}
\sup _{B_{2 \rho \varepsilon R}}\left|h_{r}\right| & \leqslant C\left(c(2 \sigma \varepsilon R)^{a} z(0)+\varepsilon^{m}(2 \rho \varepsilon R)^{2-n / p} \sum_{i=1}^{N}\left\|D^{2} z\right\|_{L^{p}\left(B_{r_{i}}\right)}\right) \\
& \leqslant C\left(c(2 \sigma \varepsilon R)^{a} z(0)+\varepsilon^{m}(2 \rho \varepsilon R)^{2-n / p} \sum_{i=1}^{N} r_{i}^{-2+n / p}\|z\|_{L^{\infty}\left(B_{2 r_{i}}\right)}\right) \\
& \leqslant C\left(c(2 \sigma \varepsilon R)^{a} z(0)+\varepsilon^{m}(2 \rho \varepsilon R)^{2-n / p} \sum_{i=1}^{N} r_{i}^{-2+n / p}\left(\frac{\rho}{\sigma}\right)^{H} z(0)\right) \\
& \leqslant C(R)\left((\sigma \varepsilon)^{a}+\varepsilon^{m} \sigma^{-\kappa}\right) z(0)
\end{aligned}
$$

where $\kappa=2+n-n / p+H$ and $H=\log C_{H}$, where $C_{H}$ is the Harnack constant. Minimizing in $\sigma$, we get

$$
\sigma_{\min }=c \varepsilon^{(m-a) /(\kappa+a)} .
$$

As a consequence, assuming $m>a$ we get

$$
\sup _{B_{2 \rho \varepsilon R}}\left|h_{r}\right| \leqslant c_{0} \varepsilon^{q_{0}} z(0)
$$

where

$$
q_{0}=\frac{a(m+\kappa)}{a+\kappa} .
$$

In $B_{2 \varepsilon R / 3}$, for $\sigma_{\min }<1 / 3$, we get, recalling the Harnack inequality and $(8)$,

$$
\begin{aligned}
z(x+\varepsilon \tau)-z(x) & =z(x+\varepsilon \tau)-u(x+\varepsilon \tau)+u(x+\varepsilon \tau)-u(x)+u(x)-z(x) \\
& \geqslant \lambda \varepsilon(u(x)-z(x))+\lambda \varepsilon z(x)-2 c_{0} \varepsilon^{q_{0}} z(0) \\
& \geqslant-c_{0} \lambda \varepsilon^{1+q_{0}} z(0)+\lambda c^{\prime} \varepsilon z(0)-2 c_{0} \varepsilon^{q_{0}} z(0) \\
& \geqslant\left(\lambda\left(c^{\prime} \varepsilon-c_{0} \epsilon^{1+q_{0}}\right)-2 c_{0} \varepsilon^{q_{0}}\right) z(0) \\
& \geqslant\left(c^{\prime \prime} \lambda \varepsilon-2 c_{0} \varepsilon^{q_{0}}\right) z(0) \geqslant c \lambda \varepsilon z(x),
\end{aligned}
$$

that is, $z$ is strictly $\varepsilon$-monotone along $\tau$ in $B_{2 \varepsilon R / 3}$ whenever

$$
q_{0} \geqslant 1+\frac{\left|\log \frac{(1-c) \lambda}{2 c_{0}}\right|}{|\log \varepsilon|} .
$$

It follows from Lemma 1 in [2] that we can choose $R=R(n)$ large enough such that

$$
D_{\tau} z(0) \geqslant c \frac{z(\varepsilon \tau)-z(0)}{\varepsilon} \geqslant c c_{1} \lambda z(0) .
$$

Now we can estimate the gradient of $h_{r}$ in $B_{\varepsilon R / 4}$. Recalling the $W^{2, p}$ estimate we have

$$
\begin{aligned}
(\varepsilon R)^{2-n / p}\left\|D^{2} h_{r}\right\|_{L^{p}\left(B_{\varepsilon} R / 4\right)} & \leqslant C\left(\left\|h_{r}\right\|_{L^{\infty}\left(B_{\varepsilon} R / 2\right)}+(\varepsilon R)^{2-n / p}\left\|f_{r}\right\|_{L^{p}\left(B_{\varepsilon} / 2 R\right)}\right) \\
& \leqslant C\left(c_{0} \varepsilon^{q_{0}} z(0)+(\varepsilon R)^{2-n / p} \varepsilon^{m}\left\|D^{2} z\right\|_{L^{p}\left(B_{\varepsilon R}\right)}\right) \\
& \leqslant C\left(c_{0} \varepsilon^{q_{0}} z(0)+\varepsilon^{m} z(0)\right) \leqslant C \varepsilon^{q_{0}} z(0) .
\end{aligned}
$$


Now by the Sobolev imbedding we get

$$
\frac{\varepsilon}{4} R \sup _{B_{\varepsilon R / 4}}\left|\nabla h_{r}\right| \leqslant C(\varepsilon R)^{2-n / p}\left\|D^{2} h_{r}\right\|_{L^{p}\left(B_{\varepsilon R / 2}\right)} \leqslant C \varepsilon^{q_{0}} z(0) .
$$

As a consequence, recalling (9), we get

$$
D_{\tau} u(0) \geqslant D_{\tau} z(0)-C \varepsilon^{q_{0}} z(0) / \varepsilon \geqslant\left(c c_{1} \lambda-C \varepsilon^{q_{0}-1}\right) z(0) .
$$

In particular, it follows from (10) and (9) that

$$
\varepsilon\left(c c_{1} \lambda+C \varepsilon^{q_{0}-1}\right) z(0) \geqslant|u(\varepsilon \tau)-u(0)| \geqslant|z(\varepsilon \tau)-z(0)|-\varepsilon \sup _{B_{\varepsilon R / 4}}\left|\nabla h_{r}\right| \geqslant \varepsilon\left(c c_{1} \lambda-C \varepsilon^{q_{0}-1}\right) z(0) .
$$

Eventually, if $q_{0}>2$ and $\varepsilon$ is small, we complete the proof, since

$$
D_{\tau} u(0) \geqslant C \frac{|u(\varepsilon \tau)-u(0)|}{\varepsilon} z(0) .
$$

We now prove that for a strictly $\varepsilon$-monotone solution $u$ of our f.b.p., at least $R \varepsilon$-away from the free boundary $\mathcal{F}_{u},|\nabla u(x)|$ behaves like $u^{+} / \operatorname{dist}\left(x, \mathcal{F}_{u}\right)$, since $u$ becomes fully monotone and its level surfaces become Lipschitz graphs.

LEMMA 3.4 Assume that $u \in C\left(\mathcal{C}_{1}\right)$ is strictly $\varepsilon$-monotone along $\Gamma\left(\vartheta, e_{n}\right)$ and $u$ is a solution of $F\left(D^{2} u(x), x\right)=0$ in $\mathcal{C}_{1}^{ \pm}(u)$. Then there exist positive numbers $\varepsilon_{0}, R, C=C(\vartheta)$ such that if $\varepsilon \leqslant \varepsilon_{0}, x \in \mathcal{C}_{1 / 2}$ and $\operatorname{dist}\left(x, \mathcal{F}_{u}\right)>C R \varepsilon$, then

$$
\frac{u(x)}{\operatorname{dist}\left(x, \mathcal{F}_{u}\right)} \sim|\nabla u(x)| .
$$

Proof. Since the proof for the two phases is similar, we give the proof only for the positive one. Let $x \in \mathcal{C}_{1}^{+}(u), d_{x}=\operatorname{dist}\left(x, \mathcal{F}_{u}\right)$. From interior estimates, we get

$$
d_{x}|\nabla u| \leqslant c_{0} u(x) .
$$

We have to prove the reverse inequality

$$
d_{x}|\nabla u| \geqslant c u(x)
$$

when $d_{x} \geqslant c R \varepsilon$. Let $x_{0} \in \mathcal{C}_{1}^{+}(u)$ be such that $d_{x_{0}}=100 L^{\prime} R \varepsilon$, and set $u\left(x_{0}\right)=b$. Notice that $\{u=b\}$ is the graph of a Lipschitz function with Lipschitz norm $L^{\prime}$, since by Lemma 3.3, $u$ is fully monotone along the directions of a cone $\Gamma\left(\vartheta, e_{n}\right)$ outside the neighborhood $\mathcal{N}_{R \varepsilon}$ of $\mathcal{F}_{u}$, and moreover the geometric arguments in Corollary 3.1 and Lemma 3.2 apply. Let $v$ be the solution of the problem

$$
\begin{cases}F\left(D^{2} v(x), x\right)=0 & \text { in } T_{\varepsilon} \\ v=1 & \text { on } \Sigma_{\varepsilon} \\ v=0 & \text { on } \partial T_{\varepsilon} \backslash \Sigma_{\varepsilon}\end{cases}
$$

where

$$
\begin{aligned}
T_{\varepsilon} & =B_{k \varepsilon}^{\prime}\left(x_{0}\right) \times\left[-200 L^{\prime} R \varepsilon, 200 L^{\prime} R \varepsilon\right], \\
\Sigma_{\varepsilon} & =\partial T_{\varepsilon} \cap\left\{x_{0 n}-20 R \varepsilon<x_{n}<20 R \varepsilon\right\}
\end{aligned}
$$


and $k=k(\vartheta)$ is properly small. By the strong maximum principle, we have

$$
b=u\left(x_{0}\right) \leqslant v\left(x_{0}\right) \max _{\Sigma_{\varepsilon}} u^{+} \equiv \gamma \max _{\Sigma_{\varepsilon}} u^{+}
$$

with $0<\gamma<1$. Then there exists a point $\bar{x} \in \partial T_{\varepsilon}$ such that $d_{x} \geqslant C L^{\prime} R \varepsilon$ for all $x \in\{u=k b\}$ $\cap B_{4 k \varepsilon}(\bar{x})$. Proceeding now as in Lemma 2.6 of [11], we obtain the result.

\section{The auxiliary perturbation function}

In this section we consider flat free boundaries. First we recall the following two results proved in [11]. Let $u: \Omega \rightarrow \mathbb{R}$ be continuous and $g$ be a positive $C^{2}$ function on $\mathbb{R}^{n}$. We define $v(x)=$ $\sup _{B_{g(x)}(x)} u$ in $\left\{x \in \Omega: B_{g(x)}(x) \subset \Omega\right\}$.

Proposition 4.1 Assume that $F$ satisfies conditions (a)-(c). Let $g$ be a positive $C^{2}$ function in $B_{1}$, with $g \leqslant m_{0}$ in $B_{1}$ ( $m_{0}$ a positive number), such that

$$
\mathcal{P}^{-}\left(D^{2} g(x)\right)-C \frac{(|\nabla g(x)|+\omega(g(x)))^{2}}{g(x)} \geqslant 0 .
$$

Let $u$ be a continuous function defined in a domain large enough so that the function

$$
v(x)=\sup _{B_{g(x)}(x)} u=\sup _{|\nu|=1} u(x+g(x) v)
$$

is well defined in $B_{1}$. Then there exist constants $\mu, \omega_{0}, c_{0}$ depending only on $\lambda, \Lambda, n$ such that if $|\nabla g|<\mu, \omega(g(x))<\omega_{0}, C>c_{0}$ and $u$ is a viscosity solution of $F\left(D^{2} u(x), x\right)=0$ in $\{u>0\}$, then $v$ is a viscosity subsolution of $F\left(D^{2} v(x), x\right)=0$ in $\{v>0\}$.

Corollary 4.1 Let $u, g$ be as in Proposition 4.1, with $g$, in particular, satisfying [11]. Let

$$
v_{\tau, g}(x):=\sup _{B_{g(x)}(x)} u(y-\tau),
$$

where $\tau \in \mathbb{R}^{n}$ is a fixed small vector. Then $v_{\tau, g}$ is a subsolution of $F\left(D^{2} u(x), x\right)=0$ in $\left\{v_{\tau, g}>0\right\}$.

To prove Theorem[1.1 (see Section 5) we follow the main ideas in [1], [2] and [13]. The strategy of the proof is based on the following main steps:

1) We improve the cone of strict $\varepsilon$-monotonicity in a half size cylinder $\mathcal{C}_{1 / 2}$ (as in Section 3 ).

2) We keep, by rescaling to $\mathcal{C}_{1}$, a proper control on the coefficients (see Lemma 3.2) and we can start reducing $\varepsilon$. As a consequence, in a slightly smaller ball, we obtain an increase in flatness along directions in a larger cone. This is what we call the basic iteration step (Lemma 4.2. Repeating the process, in the course of each iteration the constant of strict $\varepsilon$-monotonicity deteriorates at the rate of the cone opening so that, once again, a delicate balance is required between that speed and the improvement of flatness.

3) We get a geometric improvement of $\varepsilon$-monotonicity in a sequence of dyadic cylinders, along the directions of a sequence of cones whose defect angles decrease at a geometric rate (Theorems 5.1 and 5.2. This gives the final $C^{1, \gamma}$ regularity. 
We now construct a family of subsolutions that plays a decisive role in the improvement of $\varepsilon$-monotonicity. Assume that $u$ is a solution of our f.b.p. which is strictly $\varepsilon$-monotone along the directions of a cone $\Gamma\left(\vartheta, e_{n}\right)$, with $\varepsilon$ small and the defect angle $\delta=\pi / 2-\vartheta$ close to zero.

Let $R>0$ and

$$
\mathcal{N}_{R \varepsilon}=\mathcal{N}_{R \varepsilon}\left(\mathcal{F}_{u}\right)=\left\{x: \operatorname{dist}\left(x, \mathcal{F}_{u}\right)<R \varepsilon\right\} \cap \mathcal{C}_{1},
$$

the $R \varepsilon$-neighborhood of $\mathcal{F}_{u}$. Moreover, let $\Omega_{\varepsilon}$ be a smooth, flat domain such that

$$
\mathcal{N}_{R \varepsilon / 2} \subset \Omega_{R \varepsilon} \subset \mathcal{N}_{R \varepsilon}
$$

and denote by $\mathcal{F}_{\varepsilon}^{+}$the upper part of $\partial \Omega_{R \varepsilon}$, that is,

$$
\mathcal{F}_{\varepsilon}^{+}=\partial \Omega_{R \varepsilon} \cap \mathcal{C}_{1} \cap\{u>0\}
$$

Moreover, for fixed positive $\varepsilon$ and $\alpha$ we set

$$
S_{\varepsilon, \alpha}=\left\{x:\left|x^{\prime}\right|<1-\varepsilon^{\alpha}\right\}
$$

where $x=\left(x^{\prime}, x_{n}\right)$. We now construct a perturbation family of functions.

LEMMA 4.1 Let $C, c, \omega_{0}$ be positive numbers. If $C>1$ and $\omega_{0}$ is small enough, there exists a family of functions $\phi_{t}, 0 \leqslant t \leqslant 1$, such that $\phi_{t} \in C^{2}\left(\bar{\Omega}_{R \varepsilon}\right)$ and:

(a) $0<1-\omega_{0}<\phi_{t} \leqslant 1+t-\omega_{0}$,

(b) $\phi_{t} \mathcal{P}^{-}\left(D^{2} \phi_{t}\right) \geqslant C\left(\left|\nabla \phi_{t}\right|^{2}+\omega_{0}^{2}\right)$,

(c) $\phi_{t} \leqslant 1$ in $\bar{\Omega}_{R \varepsilon} \cap\left\{x: 1-\varepsilon^{\alpha} / 2<\left|x^{\prime}\right|<1\right\}$, where $0<\alpha<1$;

(d) $\phi_{t} \geqslant 1-\omega_{0}+t\left(1-c \varepsilon^{\gamma}\right)$, with $\gamma \leqslant 1-\alpha$, in $\bar{\Omega}_{R \varepsilon} \cap S_{\varepsilon, \alpha}$;

(e) $\left|\nabla \phi_{t}\right| \leqslant c \varepsilon^{-\alpha}$.

Proof. Let $\mathcal{F}_{\varepsilon}^{+}$be the upper part of $\partial \Omega_{R \varepsilon}$. Under the dilation $x \rightarrow \varepsilon, \mathcal{F}_{\varepsilon}^{+}$becomes a uniformly smooth surface $\mathcal{H}_{\varepsilon}^{+}$at a distance of order 1 from the dilated free boundary. Due to the flatness of $\mathcal{F}_{\varepsilon}$, the curvature of $\mathcal{H}_{\varepsilon}^{+}$is bounded by $c \delta$, where $\delta$ is the defect angle. Then, the distance function $d_{\varepsilon}(x)=\operatorname{dist}\left(x, \mathcal{H}_{\varepsilon}^{+}\right)$is well defined up to distance of order $1 / \delta$ and we have $\left|D_{i j} d_{\varepsilon}\right| \leqslant c \delta$.

Let $g \in C^{\infty}\left(\mathbb{R}^{+}\right)$be such that

$$
g(s)= \begin{cases}1 & \text { if } 1-\varepsilon^{\alpha} / 2 \leqslant s \leqslant 1 \\ 0 & \text { if } 0 \leqslant s \leqslant \varepsilon^{\alpha}\end{cases}
$$

We now define

$$
G(x)=g\left(\left|x^{\prime}\right|\right)+K \varepsilon^{2-\alpha}\left[1-\sigma d_{\varepsilon}\left(\frac{x}{\varepsilon}\right)\right] d_{\varepsilon}\left(\frac{x}{\varepsilon}\right),
$$

for $K>0$ and $\sigma$ to be chosen later. We have

$$
\nabla G(x)=\nabla g\left(\left|x^{\prime}\right|\right)+K \varepsilon^{1-\alpha}\left(1-2 \sigma d_{\varepsilon}\left(\frac{x}{\varepsilon}\right)\right) \nabla d_{\varepsilon}\left(\frac{x}{\varepsilon}\right)
$$

and

$$
|\nabla G(x)| \leqslant c_{1} \varepsilon^{-\alpha} .
$$


Moreover,

$$
D^{2} G(x)=D^{2} g\left(\left|x^{\prime}\right|\right)+K \varepsilon^{-\alpha}\left[\left(1-2 \sigma d_{\varepsilon}\left(\frac{x}{\varepsilon}\right)\right) D^{2} d_{\varepsilon}\left(\frac{x}{\varepsilon}\right)-2 \sigma \nabla d_{\varepsilon}\left(\frac{x}{\varepsilon}\right) \cdot \nabla d_{\varepsilon}\left(\frac{x}{\varepsilon}\right)^{T}\right] .
$$

In particular, for every $A \in \mathcal{A}_{\lambda, \Lambda}$, by properly choosing $\sigma=\sigma(\vartheta)$ and $K>0$ we have

$$
\operatorname{Tr}\left(A D^{2} G(x)\right) \leqslant-C K \varepsilon^{-\alpha} .
$$

We now define

$$
\psi(x)=\left(\frac{1+G(x)}{3}\right)^{1 /(1-2 C)}
$$

We have

$$
\begin{aligned}
\frac{1}{3} \operatorname{Tr}\left(A D^{2} G(x)\right) & =\operatorname{Tr}\left(A D^{2} \psi(x)^{1-2 C}\right) \\
& =2 C(2 C-1) \psi(x)^{-1-2 C}\langle A \nabla \psi, \nabla \psi\rangle-(2 C-1) \psi(x)^{-2 C} \operatorname{Tr}\left(A D^{2} \psi(x)\right) \\
& \leqslant-\frac{1}{3} C K \varepsilon^{-\alpha}
\end{aligned}
$$

Then

$$
\operatorname{Tr}\left(A D^{2} \psi(x)\right) \geqslant \frac{2 C \lambda|\nabla \psi(x)|^{2}}{\psi(x)}+3 C K|\nabla \psi(x)|,
$$

and as a consequence we get

$$
\mathcal{P}^{-}\left(D^{2} \psi(x)\right) \geqslant \frac{2 C \lambda|\nabla \psi(x)|^{2}}{\psi(x)}+3 C K|\nabla \psi(x)| .
$$

We now define

$$
\phi_{t}(x)=1+\omega_{0}\left(\left|x^{\prime}\right|^{2}-1\right)+t\left(\frac{\psi(x)-1}{2^{1 /(1-2 C)}-1}\right), \quad 0 \leqslant t \leqslant 1 .
$$

From (12), it is not difficult to check that the family $\phi_{t}$ has all the properties (a)-(e).

Let now

$$
v_{t}(x)=\sup _{B_{\sigma \phi_{t}(x)}(x)} u(y-\tau)=\sup _{|v|=1} u\left(x+\sigma \phi_{t}(x) v-\tau\right),
$$

where $\phi_{t}$ is the function constructed in the previous lemma, with $\varepsilon / 2<\sigma<2 \varepsilon$ and $0 \leqslant t<1$, and $\tau$ is a small vector, i.e. there exists a positive constant such that $|\tau| \leqslant c \varepsilon$. Then $v_{t}$ is well defined in $\mathcal{C}_{1-4 \varepsilon}$. We set $\Omega^{+}\left(v_{t}\right)=\mathcal{C}_{1-4 \varepsilon} \cap\left\{v_{t}>0\right\}$. If 7$\}$ holds, according to Proposition 4.1 and Corollary 4.1. $v_{t}$ is a viscosity subsolution of $F\left(D^{2} v_{t}(x), x\right)=0$ in $\left\{v_{t}>0\right\}$, and, from Lemma 3.3 (we know that $u$ is fully monotone outside an $\mathcal{N}_{R c \varepsilon}$ neighborhood), it also follows that $v_{t}$ is monotone along a cone with $|\vartheta-\bar{\vartheta}| \leqslant c \varepsilon$. In particular, the level sets of $v_{t}$ are uniformly Lipschitz graphs. This is a straightforward consequence of Lemma 2 in [2] and property (e) of Lemma 4.1. Before introducing the basic iteration step contained in the following Lemma 4.2 we remark that our operators are not linear. So we have to slightly modify the classical proof contained in [2, Lemmas 4 and 5] (see also [5, Lemma 5.7] and [12, Lemma 4.2], or [16, Lemmas 4 and 5]). 
Actually, we have to modify the family of functions containing the perturbation as we need to take in account the distortion on the free boundary. Indeed, we deal with nonlinear operators (see [2], and [13] for the linear case). Moreover, we cannot simply add a perturbation, as in [16, Lemma 4 and Remark]. More precisely, we recall that the operators considered in [16] have a particular structure (see Remark in [16]). As a consequence, in general, we cannot assume that adding a subsolution to a solution, of a given fully nonlinear operator, still yields a subsolution of the same operator. We recall that our class of operators strictly contains the fully nonlinear operators considered in [16]. Hence, we proceed by adapting the idea contained in [10, Lemma 3.1]. In particular, we define $\bar{v}_{t}$ as the solution of the problem

$$
\begin{cases}F\left(D^{2} \bar{v}_{t}(x), x\right)=0 & \text { in } \Omega_{R \varepsilon} \cap \Omega^{+}\left(v_{t}\right), \\ \bar{v}_{t}=v_{t}+A_{1} \varepsilon^{\gamma} u & \text { on } \mathcal{F}_{R \varepsilon}^{+}, \\ \bar{v}_{t}=v_{t} & \text { on } \partial\left(\Omega_{R \varepsilon} \cap \Omega^{+}\left(v_{t}\right)\right) \backslash \mathcal{F}_{R \varepsilon}^{+} .\end{cases}
$$

We extend $\bar{v}_{t}$ to $v_{t}$ in $\mathcal{C}_{1} \backslash \Omega^{+}\left(v_{t}\right)$ and, for simplicity, we still denote this new function as $\bar{v}_{t}$. Hence, $\bar{v}_{t}$ is still a viscosity subsolution of $F\left(D^{2} u(x), x\right)=0$ in $D_{R, \varepsilon}=\left(\mathcal{C}_{1} \backslash \Omega^{+}\left(v_{t}\right)\right) \cup \Omega_{R \varepsilon}$ for $0 \leqslant t \leqslant 1, A_{1}>0, \alpha<\gamma<1$. Notice that whenever $\mathcal{N}_{R \varepsilon}$ is Lipschitz, we can consider the solution of (13) to be well-defined when we take $\Omega_{R \varepsilon}=\mathcal{N}_{R \varepsilon}$.

The next result is the basic iteration step in the $\varepsilon$-monotonicity improvement.

LEMMA 4.2 Suppose that $G$ is strictly increasing in $\mathbb{R}^{+}$and assume that there exists a large positive number $N$ such that $s^{-N} G(s)$ is decreasing. Let $u$ be a solution of our f.b.p. in $\mathcal{C}_{1}$ such that $u$ is strictly $\varepsilon$-monotone along the cone of directions $\Gamma\left(\vartheta_{0}, e_{n}\right)$ with $\pi / 4<\vartheta_{0} \leqslant \pi / 2$. Then there exist $c_{0}>0$ and $0<\lambda<1$, depending on $\vartheta_{0}$, and $\varepsilon_{0}$ depending on $\vartheta_{0}$, such that if $\varepsilon \leqslant \varepsilon_{0}$ and $\vartheta_{0} \leqslant \vartheta \leqslant \pi / 2$ then $u$ is $\lambda \varepsilon$-monotone along the cone $\Gamma\left(\vartheta-c_{0} \varepsilon^{1 / 4}, e_{n}\right)$ in $\mathcal{C}_{1-\varepsilon^{1 / 8}}$ and strictly $\lambda \varepsilon$-monotone outside the $\lambda R \varepsilon$-neighborhood of $\mathcal{F}_{u}$.

Proof. Let $\lambda<1$, to be chosen later, and $u_{1}(x)=u\left(x-\lambda \varepsilon e_{n}\right)$. If $1-\lambda<\sqrt{2} / 2$, we have

$$
B_{\varepsilon(\sin \vartheta-(1-\lambda))}\left(x-\lambda \varepsilon e_{n}\right) \subset B_{\varepsilon} \sin \vartheta\left(x-\varepsilon e_{n}\right)
$$

and by the $\varepsilon$-monotonicity hypothesis,

$$
\sup _{B_{\varepsilon(\sin \vartheta-(1-\lambda))}(x)} u_{1} \leqslant u(x)
$$

in $\mathcal{C}_{1-\varepsilon^{1 / 8}} \cap \mathcal{N}_{R \varepsilon}$. Notice that, choosing a slightly smaller radius $\varepsilon\left(\sin \vartheta-\left(1-\lambda^{\prime}\right)\right), \lambda^{\prime}<\lambda$, we have strict inequality in (14). On the other hand, by Lemma 3.3, $u$ is fully monotone outside the $\mathcal{N}_{R \varepsilon}$ neighborhood of $\mathcal{F}_{u}$, therefore

$$
\sup _{B_{\lambda \varepsilon \sin \vartheta}(x)} u_{1} \leqslant u(x)
$$

for any $x \notin \mathcal{N}_{R \varepsilon}$ and for every unit vector $\tau \in \Gamma\left(\vartheta, e_{n}\right)$. To obtain the $\lambda \varepsilon$-monotonicity of $u$, first, we prove that, for a suitable $\lambda$,

$$
\sup \left\{u_{1}(y):|y-x|<\lambda \varepsilon \sin \left(\vartheta-c_{0} \varepsilon^{1 / 4}\right)\right\} \leqslant u(x) .
$$

Moreover, in $\mathcal{C}_{1-\varepsilon^{1 / 8}} \cap\left(\mathcal{N}_{R \varepsilon} \backslash \mathcal{N}_{\lambda R \varepsilon / 2}\right)$,

$$
\sup \left\{u_{1}(y):|y-x|<\lambda \varepsilon \sin \left(\vartheta-c_{0} \varepsilon^{1 / 4}\right)\right\} \leqslant u(x)-C \varepsilon^{1 / 4} u^{+}(x),
$$


so that we get strict $\lambda \varepsilon$-monotonicity of $u^{+}$in that set. To obtain our estimates, we use the family $\phi_{t}$, constructed in Lemma 4.1, to find a suitable $\bar{t}, 0<\bar{t}<1$, and a corresponding intermediate radius $\sigma \phi_{\bar{t}}$, such that

$$
\bar{v}_{\bar{t}} \leqslant u
$$

in $\mathcal{C}_{1-\varepsilon^{1 / 8}} \cap \mathcal{N}_{R \varepsilon}$ and

$$
\phi_{\bar{t}} \geqslant \lambda \sin \vartheta-\widetilde{c} \varepsilon^{1 / 4}
$$

for some $\tilde{c}>0$. Indeed, since

$$
\phi_{\bar{t}} \geqslant \lambda\left(\sin \vartheta-c_{0} \varepsilon^{1 / 4}\right) \geqslant \lambda \sin \left(\vartheta-\widetilde{c}_{0}^{1 / 4} \varepsilon\right),
$$

from the above estimates we get

$$
\sup _{B_{\lambda \varepsilon \sin \left(\vartheta-c_{0} \varepsilon^{1 / 4}\right)}(x)} u_{1} \leqslant \sup _{\left.B_{\varepsilon \lambda \sin \left(\vartheta-\tau_{0} / 4\right.} \varepsilon\right)} u_{1} \leqslant \sup _{B_{\varepsilon \phi_{\bar{t}}}(x)} u_{1} \leqslant u
$$

in $\mathcal{C}_{1-\varepsilon^{1 / 8}} \cap \mathcal{N}_{R \varepsilon}$. Since $u(x) \sim d\left(x, \mathcal{F}_{u}\right)|\nabla u(x)|$ in $\mathcal{C}_{1-\varepsilon^{1 / 8}} \cap\left(\mathcal{N}_{R \varepsilon} \backslash \mathcal{N}_{\lambda R \varepsilon / 2}\right) \cap \Omega^{+}(u)$ by Lemma 3.4. we have

$$
\sup _{B_{\lambda \varepsilon \sin \left(\vartheta-c_{0} \varepsilon^{1 / 4}\right)}(x)} u_{1} \leqslant \sup _{B_{\varepsilon \lambda \sin \left(\vartheta-\tau_{0}^{1 / 4} \varepsilon\right)}(x)} u_{1} \leqslant \sup _{B_{\varepsilon \phi_{\bar{t}}}(x)} u_{1} \leqslant u(x)-A_{1} \varepsilon^{1 / 4} u^{+}(x) .
$$

Choose $\gamma=1 / 4$ and $\sigma=\varepsilon(\sin \vartheta-(1-\lambda))$ with $\lambda \geqslant 3 / 2-1 / \sqrt{2}$. Now to select $\bar{t}$ we first make sure that for every $t \in[0, \bar{t}]$,

$$
\sigma \phi_{t} \leqslant \varepsilon\left(\lambda \sin \vartheta-\bar{c} \varepsilon^{1 / 4}\right)
$$

for some positive constant $\bar{c}$ that we will choose later. Since, keeping in mind the meaning of $\omega_{0}$ in Lemma 4.1, we have

$$
\sigma \phi_{t} \leqslant \varepsilon[\sin \vartheta-(1-\lambda)]\left(1+t-\omega_{0}\right)
$$

we require that

$$
\varepsilon[\sin \vartheta-(1-\lambda)]\left(1+t-\omega_{0}\right) \leqslant \varepsilon\left(\lambda \sin \vartheta-\bar{c} \varepsilon^{1 / 4}\right),
$$

and

$$
[\sin \vartheta-(1-\lambda)]\left(1+\bar{t}-\omega_{0}\right)=\lambda \sin \vartheta-\bar{c} \varepsilon^{1 / 4}
$$

Since

$$
\frac{\lambda \sin \vartheta-\bar{c} \varepsilon^{1 / 4}}{\sin \vartheta-(1-\lambda)} \leqslant \frac{\lambda \sin \frac{\pi}{4}}{\sin \frac{\pi}{4}-(1-\lambda)}
$$

by choosing $\lambda<1$ close enough to 1 to have

$$
\frac{\lambda \sin \frac{\pi}{4}}{\sin \frac{\pi}{4}-(1-\lambda)} \leqslant 2-\omega_{0},
$$

there exists $\bar{t} \in(0,1]$ such that 16 holds. With this choice of $\bar{t}$ we deduce that, in $\mathcal{C}_{1-\varepsilon^{1 / 2}} \cap \mathcal{N}_{R \varepsilon}$,

$$
\begin{aligned}
\sigma \phi_{\bar{t}} & \geqslant \sigma\left[1-\omega_{0}+\bar{t}\left(1-c \varepsilon^{1 / 4}\right)\right]=\varepsilon[\sin \vartheta-(1-\lambda)]\left(1-\omega_{0}+\bar{t}\left(1-c \varepsilon^{1 / 4}\right)\right) \\
& =\varepsilon\left(\lambda \sin \vartheta-\bar{c} \varepsilon^{1 / 4}\right)-c \bar{t} \varepsilon^{5 / 4} \geqslant \varepsilon\left(\lambda \sin \vartheta-c \varepsilon^{1 / 4}\right),
\end{aligned}
$$


since, in $\mathcal{C}_{1-\varepsilon^{1 / 2}} \cap \mathcal{N}_{R \varepsilon}$,

$$
\phi_{t} \geqslant 1-\omega_{0}+t\left(1-c \varepsilon^{1 / 4}\right) .
$$

In order to emphasize the comparison between $u_{1}$ and $u$ we denote $u$ as $u_{2}\left(u_{2} \equiv u\right)$. We are now ready to prove $(15)$, i.e. we verify that

$$
\bar{v}_{t} \leqslant u_{2}
$$

in $\mathcal{C}_{1-\varepsilon^{1 / 4}} \cap \mathcal{N}_{R \varepsilon}$ for every $t \in[0, \bar{t}]$. Indeed, along $\mathcal{C}_{1-\varepsilon^{1 / 4}} \cap \partial \mathcal{N}_{R \varepsilon}$, we have, for $k_{1}<k_{2} \leqslant \lambda \varepsilon \sin \vartheta$,

$$
\sup _{B_{k_{1}}(x)} u_{1} \leqslant \sup _{B_{k_{2}}(x)} u_{1}-\left(k_{2}-k_{1}\right) C\left|\nabla u_{1}\right|(x) \leqslant\left(1-\frac{k_{2}-k_{1}}{C \varepsilon}\right) u_{2},
$$

since (recalling the definition of $u_{1}$ and $u_{2}$ in terms of $u$ ), we know from Lemma 3.4 that $u(x) \sim$ $d\left(x, \mathcal{F}_{u}\right)|\nabla u(x)|$. By taking

$$
k_{1}=\lambda \varepsilon \sin \vartheta-C \varepsilon^{1+1 / 4} \quad \text { and } \quad k_{2}=\lambda \varepsilon \sin \vartheta-\frac{C}{2} \varepsilon^{1+1 / 4},
$$

(recalling $\left.(16),(\sin \vartheta-(1-\lambda))\left(1+\bar{t}-\omega_{0}\right)=\lambda \sin \vartheta-C \varepsilon^{1 / 4}\right)$, we see that, adjusting the constant $\tilde{c}$, for every $t \in[0, \bar{t}]$,

$$
v_{t} \leqslant\left(1-\widetilde{c} \varepsilon^{1 / 4}\right) u_{2}
$$

along $\mathcal{C}_{1-\varepsilon^{1 / 4}} \cap \partial \mathcal{N}_{R \varepsilon}$ and hence $\bar{v}_{t}=v_{t}+A_{1} \varepsilon^{\gamma} u \leqslant u_{2}$ there, provided we choose $A_{1} \geqslant \tilde{c}$ and $\gamma \leqslant 1 / 4$.

Along $\partial \mathcal{C}_{1-\varepsilon^{1 / 4}} \cap \mathcal{N}_{R \varepsilon}$ we have $\bar{v}_{t}=v_{t}$. Moreover, on $\partial \mathcal{C}_{1-\varepsilon^{1 / 4}} \cap \mathcal{N}_{R \varepsilon}$ (since $\varepsilon^{1 / 2}>\varepsilon$, $\phi_{t}=$ $\phi_{1}=1$ in the $\varepsilon$-neighborhood of $\partial \mathcal{C}_{1-\varepsilon^{1 / 4}} \cap \mathcal{N}_{R \varepsilon}$ ), we get $v_{t}=\sup _{B_{\sigma \phi_{t}(x)}(x)} u_{1}$, where $\left|\phi_{t}-1\right|$ is small. In particular, by abuse of notation, we write simply $v_{1}=\sup _{B_{\sigma \phi_{t}(x)}(x)} u_{1}$ whenever $\left|\phi_{t}-1\right|$ is small. Hence

$$
v_{1}(x) \leqslant u_{2}(x)
$$

and arguing as before yields

$$
v_{t}=v_{1}(x) \leqslant\left(1-C \varepsilon^{1 / 4}\right) u_{2}(x)
$$

on $\partial \mathcal{C}_{1-\varepsilon^{1 / 4}} \cap \mathcal{N}_{R \varepsilon}$. In particular, if $\omega_{0}$ is small (see the definition of $\phi_{t}$ in Lemma 4.1p there exists a $t_{0}=t_{0}\left(\omega_{0}\right)>0$ (possibly small) with $t_{0}<\bar{t}$ such that $\phi_{t_{0}}=1$ and

$$
E=\left\{t \in\left[t_{0}, \bar{t}\right]: \bar{v}_{t} \leqslant u_{2}, \mathcal{C}_{1-\varepsilon^{1 / 4}} \cap \mathcal{N}_{R \varepsilon}\right\} \neq \emptyset,
$$

because $t_{0} \in E$. The set $E$ is closed. We prove that it is also open, showing that $E=\left[t_{0}, \bar{t}\right]$. Indeed, arguing as in [5, Lemma 5.7], we prove that

$$
\Omega^{+}\left(v_{t}\right) \subset \subset \Omega^{+}(u) \cap \mathcal{C}_{1-\varepsilon^{1 / 4}} \cap \mathcal{N}_{R \varepsilon} .
$$

If not, there exists $\tilde{t} \in\left(t_{0}, \bar{t}\right) \cap E$ such that $\mathcal{F}_{v_{t}}$ and $\mathcal{F}_{u_{2}}$ have to touch each other at a regular point $x_{0}$ that is a regular point for both free boundaries. Since $\phi_{t} \geqslant 1$ in the $\varepsilon$-neighborhood of $\partial \mathcal{C}_{1-\varepsilon^{1 / 4}} \cap \mathcal{N}_{R \varepsilon}$ we must have $x_{0} \in \bar{\Omega}_{R \varepsilon} \cap S_{\varepsilon, \alpha}$ and the set $\mathcal{F}_{v_{t}}$ has at $x_{0}$ a one-sided tangent ball with center $y$ from the $\Omega^{+}\left(v_{t}\right)$ side. 
Notice that $\Omega^{+}\left(\bar{v}_{t}\right) \subset \Omega^{+}\left(u_{2}\right)$ and $\mathcal{F}_{u_{2}}$ has the same tangent ball at $x_{0}$. Moreover, $u_{2}$ is a solution of the free boundary problem, so that

$$
u_{2}(x)=\alpha_{1}\left\langle x-x_{0}, v\right\rangle^{+}-\beta_{1}\left\langle x-x_{0}, v\right\rangle^{-}+o\left(\left|x-x_{0}\right|\right)
$$

and $\alpha_{1}=G\left(\beta_{1}\right)$.

Recalling Lemma 2 in [2] or Lemma 4.10 in [5], and keeping in mind the estimate of Lemma 4.1, it follows that

$$
v_{t} \geqslant \alpha\left\langle x-x_{0}, v\right\rangle^{+}-\beta\left\langle x-x_{0}, v\right\rangle^{-}+o\left(\left|x-x_{0}\right|\right),
$$

where $\bar{v}=\frac{v+\sigma \nabla \phi_{t}}{\left|v+\sigma \nabla \phi_{t}\right|}, \alpha=\alpha_{1}\left|v+\sigma \nabla \phi_{t}\right|, \beta=\beta_{1}\left|\nu+\sigma \nabla \phi_{t}\right|$, and

$$
\frac{\alpha}{1-\sigma\left|\nabla \phi_{t}\right|} \geqslant G\left(\frac{\beta}{1+\sigma\left|\nabla \phi_{t}\right|}\right)
$$

Notice that

$$
\frac{\bar{v}_{t}(x)-v_{t}(x)}{v_{t}(x)} \geqslant \frac{\bar{v}_{t}(x)-\tilde{v}_{t}(x)}{\tilde{v}_{t}(x)},
$$

where

$$
\begin{cases}F\left(D^{2} \tilde{v}_{t}(x), x\right)=0 & \text { in } D_{R, \varepsilon}, \\ \tilde{v}_{t}=\bar{v}_{t} & \text { on } \partial D_{R, \varepsilon} .\end{cases}
$$

Moreover, $\mathcal{F}_{\bar{v}_{t}}$ are equi-Lipschitz with $\operatorname{Lip}\left(\mathcal{F}_{\bar{v}_{t}}\right) \leqslant L+C \varepsilon$ and $\mathcal{N}_{R \varepsilon / 2} \subset \Omega_{R \varepsilon} \subset \mathcal{N}_{R \varepsilon} \subset \Omega^{+}\left(\bar{v}_{t}\right)$. Hence by the boundary Harnack comparison principle (see Lemma 2.1) and remarking that $\bar{v}_{t}(x)-$ $\tilde{v}_{t}(x)$ is a positive supersolution of $F\left(D^{2} u, x\right)=0$, and thus a supersolution of $\mathcal{P}^{-}\left(D^{2} u\right)=0$, we deduce that there exists a positive constant $C^{\prime}$ independent of $t$ such that

$$
\frac{\bar{v}_{t}(x)-v_{t}(x)}{v_{t}(x)} \geqslant C^{\prime} A_{1} \varepsilon^{\gamma}>0 .
$$

It follows from (18) and (19) that

$$
\bar{v}_{t}(x) \geqslant \bar{\alpha}\left\langle x-x_{0}, v\right\rangle^{+}-\bar{\beta}\left\langle x-x_{0}, v\right\rangle^{-}+o\left(\left|x-x_{0}\right|\right),
$$

where $\bar{\alpha} \geqslant\left(1+C A_{1} \varepsilon^{\gamma}\right) \alpha$ and $\bar{\beta} \leqslant \beta$.

We want that

$$
\bar{\alpha} \geqslant G(\bar{\beta})
$$

Notice that, recalling (e) in Lemma 4.1 .

$$
\sigma\left|\nabla \phi_{t}\right| \leqslant c \sigma \varepsilon^{-\alpha}
$$

Thus we get

$$
\bar{\alpha} \geqslant\left(1+C A_{1} \varepsilon^{\gamma}\right)\left(1-c \sigma\left|\nabla \phi_{t}\right|\right) \alpha_{1} \geqslant\left(1+C A_{1} \varepsilon^{\gamma}\right)\left(1-c \sigma \varepsilon^{-\alpha}\right) \alpha_{1}
$$

and

$$
\bar{\beta} \leqslant\left(1+C \sigma \varepsilon^{-\alpha}\right) \beta_{1} .
$$


Moreover, $G$ is increasing. Hence inequality (20) will be satisfied if

$$
\left(1+C A_{1} \varepsilon^{\gamma}\right)\left(1-c \sigma \varepsilon^{-\alpha}\right) \alpha_{1} \geqslant G\left(\left(1+C \sigma \varepsilon^{-\alpha}\right) \beta_{1}\right)
$$

in particular if

$$
\left(1+C A_{1} \varepsilon^{\gamma}\right)\left(1-c \sigma \varepsilon^{-\alpha}\right) G\left(\beta_{1}\right) \geqslant G\left(\left(1+C \sigma \varepsilon^{-\alpha}\right) \beta_{1}\right) .
$$

On the other hand, $s^{-N} G(s)$ is decreasing. Hence 21 will be satisfied if

$$
\left(1+C A_{1} \varepsilon^{\gamma}\right)\left(1-c \sigma \varepsilon^{-\alpha}\right) \geqslant \beta_{1}^{-N}\left(\left(1+C \sigma \varepsilon^{-\alpha}\right) \beta_{1}\right)^{N},
$$

and in particular when

$$
\left(1+C A_{1} \varepsilon^{\gamma}\right)\left(1-c \sigma \varepsilon^{-\alpha}\right) \geqslant\left(1+C \sigma \varepsilon^{-\alpha}\right)^{N} .
$$

Hence, if we assume that $\sigma \leqslant \varepsilon^{q}, 0<\alpha \leqslant q \leqslant 1$ and take $A_{1}$ large enough, 23 will be satisfied and (20) holds.

This produces a contradiction with the free boundary conditions and Hopf's lemma; for the remaining details see Lemma 4.12 in [5] or Lemma 3.1 in [10].

Now, $\bar{v}_{t} \geqslant v_{t}$ on $\mathcal{C}_{1-C \varepsilon^{1 / 8}}$. Also, on such a domain, $\phi_{t}$ can be estimated from below by $1-\omega_{0}+$ $t\left(1-C \varepsilon^{1 / 4}\right)$ by construction of $\phi_{t}$. For maximal possible $t, \sigma \phi_{t} \geqslant \lambda \varepsilon \sin \vartheta-C \varepsilon^{1+1 / 4}$, that is,

$$
\sup _{\left.B_{\varepsilon(\lambda \sin \vartheta-\widetilde{c} \varepsilon} 1 / 4\right)}(x)=u_{1} \leqslant u_{2}(x) .
$$

Since

$$
\lambda \sin \vartheta-\widetilde{c} \varepsilon^{1 / 4} \geqslant \lambda \sin \left(\vartheta-c_{0} \varepsilon^{1 / 4}\right)
$$

for a suitable positive $c_{0}$, the proof is complete.

Notice that $u^{-}$satisfies analogous estimates. Indeed, it is enough to remark that $u^{-}$is a solution of $\tilde{F}\left(D^{2} u^{-}(x), x\right)=0$ in $\Omega^{-}(u)$ where $\tilde{F}(M, x)=F(-M, x)$. Notice that $\tilde{F}(M, x)$ still satisfies all our hypotheses, and previous arguments apply.

\section{Strict $\varepsilon$-monotonicity implies $C^{1, \gamma}$}

Using a double iterative argument based on Lemma 4.4 of [11] and Lemma 4.2, we can prove that strict $\varepsilon$-monotonicity implies $C^{1, \gamma}$.

THEOREM 5.1 Let $u$ be a solution of our f.b.p. in $\mathcal{C}_{1}$. Suppose $G$ is Lipschitz continuous, strictly increasing and that $z^{-N} G(z)$ is decreasing in $(0,+\infty)$ for some $N>0$. Let $\pi / 4<\vartheta_{0} \leqslant \pi / 2$ be given. There exist $\varepsilon_{0}=\varepsilon_{0}\left(\vartheta_{0}\right)$ such that if $u$ is strictly $\varepsilon$-monotone along the cone of directions $\Gamma\left(\vartheta, e_{n}\right)$, for some $\varepsilon \leqslant \varepsilon_{0}$ and $\vartheta \geqslant \vartheta_{0}$, then, in $\mathcal{C}_{1 / 3}, \mathcal{F}_{u}$ is the graph of a $C^{1, \gamma}$ function with $\gamma=\gamma\left(n, a, \bar{C}, \lambda, \Lambda, \vartheta_{0}, N\right)$.

Proof. Lemma 3.4 gives full monotonicity for $u, R \varepsilon$-away from $\mathcal{F}_{u}$. Proceeding now as in Lemma 4.4 of [11], we conclude the proof.

We now prove the following intermediate result. 
THEOREM 5.2 Let $u$ be a solution of our f.b.p. in $\mathcal{C}_{1}$, and assume that hypotheses (i) and (ii) of Theorem 1.1 hold. Then there exist $\vartheta_{0}<\pi / 2$ and $\varepsilon_{0}>0$, both depending on $n, a, \bar{C}, \alpha_{0}, \alpha_{1}, \lambda, \Lambda, N$, such that if $u^{+}$is strictly $\varepsilon$-monotone along any directions in $\Gamma\left(\vartheta_{0}, e_{n}\right)$ for some $\varepsilon<\varepsilon_{0}$, then $\mathcal{F}_{u}$ is the graph of a $C^{1, \gamma}$ function in $\mathcal{C}_{1 / 2}$ with $\gamma=\gamma\left(n, a, \bar{C}, \alpha_{0}, \alpha_{1}, \lambda, \Lambda, N\right)$.

The proof of Theorem 5.2 is based on the so called dichotomy Lemma 5.1] We follow the same approach as in [13].

Lemma 5.1 (Dichotomy Lemma) Assume that (ii) in Theorem 1.1 holds. Let $u$ be a solution of our f.b.p. in $\mathcal{C}_{1}$, and let $u_{\max }=\max _{\mathcal{C}_{1}}|u|$. There exist $\vartheta_{0}$ and $\varepsilon_{0}$ such that if $\pi / 4<\vartheta_{1} \leqslant \vartheta_{0} \leqslant \vartheta$ and $\varepsilon \leqslant \varepsilon_{0}$, the following alternative holds: there are constants $K$ (large) and $p>0, \eta>0$, $0<\tau_{2}<\tau_{1}<1$ such that:

(a) if $u^{-}\left(-\frac{1}{2} e_{n}\right) \geqslant K \varepsilon^{1 / 2} u_{\max }$, then $u$ is strictly $\varepsilon^{p}$-monotone along the cone $\Gamma\left(\vartheta_{1}, e_{n}\right)$ in the $\eta$-neighborhood of $\mathcal{F}_{u}$ in $\mathcal{C}_{1 / 2}$;

(b) if $u^{-}\left(-\frac{1}{2} e_{n}\right) \leqslant K \varepsilon^{1 / 2} u_{\max }$, then $u^{+}$is strictly $\lambda \varepsilon$-monotone, for some $\lambda\left(\vartheta_{0}\right)<1$, along the cone $\Gamma\left(\vartheta-\varepsilon^{\tau_{1}}, e_{n}\right)$ in $\mathcal{C}_{1-\varepsilon^{\tau_{2}}}$, where $0<\tau_{2}<\tau_{1}<1$.

Proof. Assuming we are in case (a), denote by $\mathcal{G}$ the graph of the Lipschitz function $x_{n}=h\left(x^{\prime}\right)$, with Lipschitz norm

$$
L^{\prime} \leqslant \tan (\pi / 2-\vartheta)
$$

(see Corollary 3.1 and Lemma 3.3, whose $\mathcal{N}_{\varepsilon}$ neighborhood contains $\mathcal{F}_{u}$. Let

$$
\mathcal{G}_{\varepsilon}=\left\{x_{n}=h\left(x^{\prime}\right)+2 \varepsilon\right\} \quad \text { and } \quad T_{\varepsilon}=\left\{x_{n}<h\left(x^{\prime}\right)+2 \varepsilon\right\} .
$$

The function $u^{-}$is a viscosity solution of $\bar{F}\left(D^{2} u^{-}, x\right)=0$ in $\Omega^{-}(u)$, where $\bar{F}(M, x)=$ $-F(-M, x)$, and $\bar{F}$ still belongs to our class of operators. Moreover, let $v, \widetilde{v}$ be the solutions of the Dirichlet problems

$$
\begin{cases}\bar{F}\left(D^{2} v(x), x\right)=0 & \text { in } \mathcal{C}_{7 / 8} \cap T_{0}, \\ v=0 & \text { on } \mathcal{G}, \\ v=u^{-} & \text {on } \partial\left(\mathcal{C}_{7 / 8} \cap T_{0}\right) \backslash \mathcal{G},\end{cases}
$$

and

$$
\begin{cases}\bar{F}\left(D^{2} \widetilde{v}(x), x\right)=0 & \text { in } \mathcal{C}_{7 / 8} \cap T_{2 \varepsilon}, \\ \widetilde{v}=0 & \text { on } \mathcal{G}_{2 \varepsilon}, \\ \widetilde{v}=u^{-} & \text {on } 2\left(\mathcal{C}_{7 / 8} \cap T_{2 \varepsilon}\right) \backslash \mathcal{G}_{2 \varepsilon}\end{cases}
$$

respectively. By the Aleksandrov-Bakelman-Pucci maximum principle, we have

$$
v \leqslant u^{-} \quad \text { in } \mathcal{C}_{7 / 8} \cap T_{0},
$$

and

$$
\tilde{v} \geqslant u^{-} \quad \text { in } \mathcal{C}_{7 / 8} \cap T_{2 \varepsilon},
$$

because $u^{-}$is a subsolution in $\mathcal{C}_{7 / 8} \cap T_{2 \varepsilon}$.

Since $\mathcal{G}$ is a Lipschitz graph, from Lemma 2.8 in [11], there exists a positive number $\eta=$ $\eta\left(L^{\prime}, n\right)$ such that in the $\mathcal{N}_{\eta}\left(\mathcal{F}_{u}\right)$ neighborhood of $\mathcal{F}_{u}, D_{\tau} u(x) \geqslant 0$ for every $\tau \in \Gamma\left(\vartheta_{0}, e_{n}\right)$. The maximum principle gives, along $\mathcal{F}_{u}$,

$$
\widetilde{v} \leqslant C \varepsilon^{a} u_{\max }
$$


We can now write

$$
v \leqslant u^{-} \leqslant \widetilde{v} \leqslant v+C \varepsilon^{a} u_{\max }
$$

For $\vartheta_{1}<\vartheta_{0}$

$$
v(x) \geqslant\left|x-x_{0}\right|^{\beta_{0}} u^{-}\left(-\frac{1}{2} e_{n}\right)
$$

for $x-x_{0} \in \Gamma\left(\vartheta_{1},-e_{n}\right)$ and $a \leqslant \beta_{0}$. Take $x_{1}, x_{2}$ in $\mathcal{N}_{\eta}(\Gamma)$ with

$$
C_{1} \varepsilon^{p} \leqslant\left|x_{1}-x_{2}\right| \leqslant C_{2} \varepsilon^{p}
$$

and $x_{1}-x_{2} \in \Gamma\left(\vartheta_{1},-e_{n}\right), p>0$ small; we want to show

$$
u^{-}\left(x_{2}\right)-u^{-}\left(x_{1}\right) \geqslant c \varepsilon^{\beta_{1}} u^{-}\left(x_{1}\right)
$$

for a suitable $\beta_{1}$. Choose $\vartheta_{0}$ such that $\vartheta_{1} \leqslant \vartheta_{0}-\pi / 8$. If $x_{1} \in \Omega^{+}(u)$, then 27) is trivial. We restrict $x_{1}$ to be below $\{y=g(x)+\varepsilon\}$ (so $x_{2}$ is far below $\mathcal{G}$ ). From Lemma 2.8 in [11] we have

$$
D_{\tau} v \geqslant 0
$$

where

$$
\tau=\frac{x_{2}-x_{1}}{\left|x_{2}-x_{1}\right|}
$$

and

$$
D_{\tau} v \geqslant C \frac{v\left(x_{2}\right)}{\delta_{0}} \quad \text { for } x-x_{1}=\lambda\left(x_{2}-x_{1}\right) \text { with } \lambda \in[1 / 2,1] .
$$

Then, by 25] and hypothesis (a), we have

$$
v\left(x_{2}\right)-v\left(x_{1}\right) \geqslant C \frac{v\left(x_{2}\right)}{\delta_{0}} \varepsilon^{p} \geqslant \frac{C}{\delta_{0}} u^{-}\left(-\frac{1}{2} e_{n}\right) \varepsilon^{\left(\beta_{0}+1\right) p} \geqslant \frac{C K}{\delta_{0}} \varepsilon^{\left(\beta_{0}+1\right) p+1 / 2} u_{\max },
$$

so, by 24), 25] and (28), we obtain

$$
\begin{aligned}
u^{-}\left(x_{2}\right)-u^{-}\left(x_{1}\right) & \geqslant v\left(x_{2}\right)-\widetilde{v}\left(x_{1}\right) \geqslant v\left(x_{2}\right)-v\left(x_{1}\right)-C \varepsilon^{a} u_{\max } \\
& \geqslant\left[\frac{C K}{\delta_{0}} \varepsilon^{\left(\beta_{0}+1\right) p+1 / 2}-C \varepsilon^{a}\right] u_{\max } \geqslant C \varepsilon^{\beta_{1}} u^{-}\left(x_{1}\right)
\end{aligned}
$$

by taking $\beta_{1}=\left(\beta_{0}+1\right) p+1 / 2<a$, where the constant $C$ depends on $\delta_{0}$ in the third equation. This concludes the proof in case (a).

Assume now (b) occurs. Here we take $u_{1}=u^{+}\left(x-\lambda \varepsilon e_{n}\right)$ and $u_{2}=u^{+}$and we argue as in Lemma 4.2

In particular (see $(13)$ ), we define $\bar{v}_{t}$ starting from $u^{+}$, with $\gamma$ to be chosen later. We want to show that, for some $\bar{t}$ in Lemma $4.2, \bar{v}_{t} \leqslant u_{2}$. It is enough to show that, for any $0 \leqslant t_{0}<t \leqslant \bar{t}$, the function $\bar{v}_{\bar{t}}$ is a subsolution at every point $x_{0} \in \mathcal{F}_{u_{2}} \cap \mathcal{F}_{\bar{v}_{t}} \cap \mathcal{C}_{7 / 8}$. We argue as in Lemma 4.2 , by contradiction. Precisely, at $x_{0}$ there is a touching ball from the left to $\mathcal{F}_{u_{2}}$, so that near $x_{0}$, nontangentially,

$$
u(x)=\alpha_{1}\left\langle x-x_{0}, v\right\rangle^{+}-\beta_{1}\left\langle x-x_{0}, v\right\rangle^{-}+o\left(\left|x-x_{0}\right|\right)
$$


with $\alpha_{1} \geqslant G\left(\beta_{1}\right)$. Therefore, at $x_{0}, \bar{v}_{t}$ satisfies the following asymptotic inequality:

$$
\bar{v}_{t}(x) \geqslant \bar{\alpha}\left\langle x-x_{0}, v\right\rangle^{+}-\beta\left\langle x-x_{0}, v\right\rangle^{-}+o\left(\left|x-x_{0}\right|\right),
$$

where (recalling that by (e) in Lemma $4.1, \sigma\left|\nabla \phi_{t}\right| \leqslant c \sigma \varepsilon^{-\alpha}$ ),

$$
\begin{aligned}
\bar{\alpha} & \geqslant\left(1+C A_{1} \varepsilon^{\gamma}\right) \alpha_{1}\left|\nu+\sigma \nabla \phi_{t}\right| \geqslant\left(1+C A_{1} \varepsilon^{\gamma}\right)\left(1-C \sigma\left|\nabla \phi_{t}\right|\right) G\left(\beta_{1}\right) \\
& \geqslant\left(1+C A_{1} \varepsilon^{\gamma}\right)\left(1-c \varepsilon^{q-\alpha}\right) G\left(\beta_{1}\right)
\end{aligned}
$$

and, by 18$]$,

$$
\frac{\alpha}{1-\sigma\left|\nabla \phi_{t}\right|} \geqslant G\left(\frac{\beta}{1+\sigma\left|\nabla \phi_{t}\right|}\right) .
$$

For simplicity assume that $q-\alpha=1 / 2$. Now if $\bar{v}_{t} \leqslant u_{2}$ we deduce that $\alpha_{1} \leqslant \bar{\alpha}$ and $\beta_{1} \geqslant \beta$. Moreover, since $G$ is increasing, we get

$$
\bar{\alpha} \geqslant\left(1+C A_{1} \varepsilon^{\gamma}\right)\left(1-c \varepsilon^{1 / 2}\right) G\left(\beta_{1}\right) \geqslant\left(1+C \varepsilon^{\gamma}\right)\left(1-c \varepsilon^{1 / 2}\right) G(0) .
$$

Thus, $\bar{v}_{t}$ will satisfy the condition on the free boundary and $\bar{v}_{t}$ will indeed be a subsolution whenever

$$
\left(1+C \varepsilon^{\gamma}\right)\left(1-c \varepsilon^{1 / 2}\right) G(0) \geqslant G(\beta) .
$$

Now, proceeding as in the Claim of Lemma 7 in [16], by using a barrier argument, we can show $\beta_{1} \leqslant c \varepsilon^{\delta}$ with $\delta>0$ small. In particular, inequality $(30)$ will be satisfied if

$$
\left(1+C \varepsilon^{\gamma}\right)\left(1-c \varepsilon^{1 / 2}\right) G(0) \geqslant G\left(c \varepsilon^{\delta}\right),
$$

because $G$ is increasing. Notice moreover that we have assumed that $G$ is Lipschitz. Hence $G\left(c \varepsilon^{\delta}\right) \leqslant G(0)+C_{1} \varepsilon^{\delta}$. As a consequence, recalling once more that $G$ is increasing, inequality (30) will be satisfied whenever

$$
\left(1+C \varepsilon^{\gamma}\right)\left(1-c \varepsilon^{1 / 2}\right) G(0) \geqslant G(0)+C_{1} \varepsilon^{\delta} .
$$

In particular, we must require that

$$
C G(0) \varepsilon^{\gamma} \geqslant C_{1} \varepsilon^{\delta}
$$

and $\gamma<\delta$. Then we get a contradiction by Hopf's lemma, and the proof proceeds as in Lemma 4.2 .

Proof of Theorem 5.2. As noted in [13], the proof reduces to Theorem 5.1] through Lemma 5.1.

Finally, we are in a position to prove the main theorem.

Proof of Theorem 1.1. We show that $u^{+}$is stricly $\varepsilon$-monotone in an $\eta$-neighborhood of $\mathcal{F}_{u}$, along a cone $\Gamma\left(\vartheta^{*}, e_{n}\right)$ with $\vartheta^{*}$ slightly smaller than $\bar{\vartheta}$. Then we apply Theorem 5.2. Let

$$
\mathcal{G}=\left\{x_{n}=h\left(x^{\prime}\right)-c_{0} \varepsilon\right\}
$$

with $c_{0}$ such that $\Omega^{+}(u) \subset\left\{x_{n}>h\left(x^{\prime}\right)-c_{0} \varepsilon\right\}$, and let $v$ be the solution of the problem

$$
\begin{cases}F\left(D^{2} v(x), x\right)=0 & \text { in }\left\{x_{n}>h\left(x^{\prime}\right)-c_{0} \varepsilon\right\}, \\ v=0 & \text { in } \mathcal{G}, \\ v=u^{+} & \text {on } \partial \mathcal{C}_{1} \cap\left\{x_{n}>h\left(x^{\prime}\right)-c_{0} \varepsilon\right\} .\end{cases}
$$


Then in $\Omega^{+}(u)$ we have

$$
u^{+}(x)+2 c_{0} \varepsilon \geqslant v(x) \geqslant u^{+}(x) \geqslant \alpha_{0} \operatorname{dist}\left(x, \mathcal{F}_{u}\right) \geqslant c_{1} \operatorname{dist}(x, \mathcal{G})-c_{2} \varepsilon .
$$

It follows from Lemma 3.1 (see also Theorem 2.2 in [11]) that $v$ is monotone increasing along a cone $\Gamma\left(\vartheta^{*}, e_{n}\right)$, with $\vartheta_{0}<\vartheta^{*}<\bar{\vartheta}$ (notice that we have to start from a large cone in order to apply Theorem 5.2 - this justifies the hypothesis on the f.b. $\mathcal{F}_{u}$; here $\bar{\vartheta}$ is the same as in Theorem 5.2), in the $\eta$-neighborhood $\mathcal{N}_{\eta} \cap \mathcal{C}_{3 / 4}$ of $\mathcal{F}_{u}$, and

$$
D_{\tau} v(x) \sim \frac{v(x)}{\operatorname{dist}(x, \mathcal{G})} \quad \text { for every } \tau \in \Gamma\left(\vartheta^{*}, e_{n}\right) .
$$

Thus, if $\eta \geqslant d(x, \mathcal{G})>c_{3} \varepsilon$, we get

$$
D_{\tau} v(x) \geqslant c_{1}-\frac{c_{2} \varepsilon}{\operatorname{dist}(x, \mathcal{G})}>\frac{1}{2} c_{1}
$$

as long as $c_{3}>2 c_{2} / c_{1}$. Therefore, in $\mathcal{N}_{\eta} \cap \mathcal{C}_{3 / 4} \cap \Omega^{+}(u)$,

$$
\begin{aligned}
u^{+}\left(x+c_{4} \varepsilon \tau\right)-u^{+}(x) & \geqslant v\left(x+c_{4} \varepsilon \tau\right)-v(x)-2 c_{0} \varepsilon=\int_{0}^{c_{4} \varepsilon} D_{\tau} v(x+s \tau) d s-2 c_{0} \varepsilon \\
& \geqslant \int_{c_{3} \varepsilon}^{c_{4} \varepsilon} D_{\tau} v(x+s \tau) d s-2 c_{0} \varepsilon \geqslant c\left(c_{4}-c_{3}\right) \varepsilon-2 c_{0} \varepsilon \geqslant C \varepsilon
\end{aligned}
$$

provided $c_{4}$ is large enough. The proof of Theorem 1.1 is complete.

\section{Acknowledgements}

The authors wish to thank Sandro Salsa for some fruitful discussions on the subject.

\section{REFERENCES}

1. CAffarelli, L. A Harnack inequality approach to the regularity of free boundaries, Part 1: Lipschitz free boundaries are $C_{\alpha}^{1}$. Rev. Mat. Iberoamer. 3 (1987), 139-162. Zbl 0676.35085 MR 0990856

2. CAfFarelli, L. A Harnack inequality approach to the regularity of free boundaries. II. Flat free boundaries are Lipschitz. Comm. Pure Appl. Math. 42 (1989), 55-78. Zbl 0676.35086 MR 0973745

3. Caffarelli, L., \& Cabré, X. Fully Nonlinear Elliptic Equations. Amer. Math. Soc. Colloq. Publ. 43, Amer. Math. Soc., Providence, RI (1995). Zbl 0834.35002 MR 1351007

4. Caffarelli, L., Crandall, M. G., Kocan, M., \& Święch, A. On viscosity solutions of fully nonlinear equations with measurable ingredients. Comm. Pure Appl. Math. 49 (1996), 365-397. Zbl 0854.35032 MR 1376656

5. Caffarelli, L., \& Salsa, S. A Geometric Approach to Free Boundary Problems. Grad. Stud. Math. 68, Amer. Math. Soc., Providence, RI (2005). Zbl 1083.35001 MR 2145284

6. Cerutti, M. C., Ferrari, F., \& SAlsa, S. Two-phase problems for linear elliptic operators with variable coefficients: Lipschitz free boundaries are $C^{1, \gamma}$. Arch. Ration. Mech. Anal. 171 (2004), 329-348. Zbl 1106.35144 MR 2038343

7. EsCAURIAZA, L. $W^{2, n}$ a priori estimates for solutions to fully nonlinear equations. Indiana Univ. Math. J. 42 (1993), 413-423. Zbl 0792.35020 MR 1237053 
8. Fabes, E., Garofalo, N., Marin-Malave, S., \& Salsa, S. Fatou theorems for some nonlinear elliptic equations. Rev. Mat. Iberoamer. 4 (1988). 227-251. Zbl 0703.35058 MR 1028741

9. Feldman, M. Regularity for nonisotropic two-phase problems with Lipschitz free boundaries. Differential Integral Equations 10 (1997), 1171-1179. Z Zbl 0940.35047 MR 1608061

10. Feldman, M. Regularity of Lipschitz free boundaries in two-phase problems for fully nonlinear elliptic equations. Indiana Univ. Math. J. 50 (2001), 1171-1200. Zbl 1037.35104 MR 1871352

11. Ferrari, F. Two-phase problems for a class of fully nonlinear elliptic operators. Lipschitz free boundaries are $C^{1, \gamma}$. Amer. J. Math. 128 (2006), 541-571. Zbl 1142.35108 MR 2230916

12. Ferrari, F., \& SAlsa, S. Subsolutions of elliptic operators in divergence form and application to two-phase free boundary problems. Bound. Value Probl. 2007, art. ID 57049, 21 pp. Zbl pre05237143 MR 2291927

13. Ferrari, F., \& SAlSA, S. Regularity of the free boundary in two-phase problems for linear elliptic operators. Adv. Math. 214 (2007), 288-322. Zbl pre05180273 MR 2348032

14. ŚwIĘCH, A. $W^{1, p}$-interior estimates for solutions of fully nonlinear, uniformly elliptic equations. $A d v$. Differential Equations 2 (1997), 1005-1027. Zbl 1023.35509 MR 1606359

15. WANG, P. Y. Regularity of free boundaries of two-phase problems for fully nonlinear elliptic equations of second order. I. Lipschitz free boundaries are $C^{1, \alpha}$. Comm. Pure Appl. Math. 53 (2000), 799-810. Zbl 1040.35158 MR 1752439

16. WANG, P. Y. Regularity of free boundaries of two-phase problems for fully nonlinear elliptic equations of second order. II. Flat free boundaries are Lipschitz. Comm. Partial Differential Equations 27 (2002), 1497-1514. Zbl 1125.35424 MR 1924475 